\title{
Editorial
}

\section{Recent Revolution in Image Technology for the Wrist}

\author{
Toshiyasu Nakamura, MD, PhD ${ }^{1}$ \\ ${ }^{1}$ Department of Orthopaedic Surgery, School of Medicine, \\ International University of Health and Welfare, Tokyo, Japan \\ J Wrist Surg 2018;7:273.
}

Innovation in diagnostic tools for wrist surgery has been remarkable in this decade. When I started treatment of the wrist in the late 1980s, magnetic resonance imaging (MRI) or ultrasound showed sand storm-like images. Arthrography served as an important diagnostic technique for triangular fibrocartilage complex (TFCC) lesions then. In the early 1990s, I performed high-resolution MRI of the wrist using small surface coil made of copper but the quality of the image was suboptimal. Now, we have MRIs of 3T or more, noninvasive high-quality ultrasound, and threedimensional computed tomography (3D-CT) that can precisely delineate tendons. These innovations are a result of the technological developments by manufacturing companies. However, static imaging has limitations to completely understand the joint, as the objective is to know normal and abnormal kinematics of the wrist. Computer simulation analysis of the carpal bones is beneficial as it shows actual joint motion in patients. Recent four-dimensional (4D)-CT delineates actual motion of the bones during joint motion. But what about soft tissues? Ultrasound can demonstrate motion of the soft tissues, whereas bone indicates the acoustic shadow field.

This issue includes the "Special Review" article "Cine MRI of the Triangular Fibrocartilage Complex during Radial-Ulnar Deviation." The authors describe that cineMRI can demonstrate behavior of the TFCC during radial and ulnar deviation. Please look at the videos on the Journal website that demonstrate normal changes in the shape of the TFCC and abnormal behavior of the TFCC in the patients. Interesting articles on wrist treatment, such as the ulnar shortening, scaphoid fracture, TFCC lesion, scapholunate ligament lesion in distal radius fracture, wrist biomechanics, and survey for scaphoid proximal pole fracture, are also included in this issue. Don't miss it.
Address for correspondence Toshiyasu Nakamura, MD, PhD, Department of Orthopaedic Surgery, School of Medicine, International University of Health and Welfare, 8-10-16 Akasaka, Minato-ku, Tokyo 107-0052, Japan (e-mail: toshiyasu@ae.em-net.ne.jp).
Copyright (c 2018 by Thieme Medical Publishers, Inc., 333 Seventh Avenue, New York, NY 10001, USA. Tel: +1(212) 584-4662.
DOI https://doi.org/ 10.1055/s-0038-1669462. ISSN 2163-3916. 